\title{
Facilitating organisational development using a group-based formative assessment and benchmarking method: design and implementation of the International Family Practice Maturity Matrix
}

Glyn Elwyn, ${ }^{1}$ Marie-Jet Bekkers, ${ }^{1}$ Laura Tapp, ${ }^{2}$ Adrian Edwards, ${ }^{1}$ Robert Newcombe, ${ }^{1}$ Tina Eriksson, ${ }^{3}$ Jozé Braspenning, ${ }^{4}$ Christine Kuch, ${ }^{5}$ Zlata Ozvacic Adzic, ${ }^{6}$ Olayinka Ayankogbe, ${ }^{7}$ Tatjana Cvetko, ${ }^{8}$ Kees in 't Veld, ${ }^{9}$ Antonis Karotsis, ${ }^{10}$ Janko Kersnik, ${ }^{8}$ Luc Lefebvre, ${ }^{11}$ llir Mecini, ${ }^{12}$ Goranka Petricek, ${ }^{6}$ Luis Pisco, ${ }^{13}$ Janecke Thesen, ${ }^{14}$ José María Turón, ${ }^{15}$ Edward van Rossen, ${ }^{16}$ Richard Grol ${ }^{4}$

For numbered affiliations see end of article.

\section{Correspondence to} Professor Glyn Elwyn, Department of Primary Care and Public Health, School of Medicine, Cardiff University, Neuadd Meirionnydd, Heath Park, Cardiff CF14 4YS, UK: elwyng@cardiff.ac.uk

Accepted 23 February 2010 Published Online First 28 May 2010

\section{ABSTRACT}

Introduction Well-organised practices deliver higherquality care. Yet there has been very little effort so far to help primary care organisations achieve higher levels of team performance and to help them identify and prioritise areas where quality improvement efforts should be concentrated. No attempt at all has been made to achieve a method which would be capable of providing comparisons-and the stimulus for further improvement-at an international level.

Methods The development of the International Family Practice Maturity Matrix took place in three phases: (1) selection and refinement of organisational dimensions;

(2) development of incremental scales based on a recognised theoretical framework; and (3) testing the feasibility of the approach on an international basis, including generation of an automated web-based benchmarking system.

Results This work has demonstrated the feasibility of developing an organisational assessment tool for primary care organisations that is sufficiently generic to cross international borders and is applicable across a diverse range of health settings, from state-organised systems to insurer-based health economies. It proved possible to introduce this assessment method in 11 countries in Europe and one in Africa, and to generate comparison benchmarks based on the data collected. The evaluation of the assessment process was uniformly positive with the view that the approach efficiently enables the identification of priorities for organisational development and quality improvement at the same time as motivating change by virtue of the group dynamics.

Conclusions We are not aware of any other organisational assessment method for primary care which has been 'born international,' and that has involved attention to theory, dimension selection and item refinement. The principal aims were to achieve an organisational assessment which gains added value by using interaction, engagement comparative benchmarks: aims which have been achieved. The next step is to achieve wider implementation and to ensure that those who undertake the assessment method ensure linkages are made to planned investment in organisational development and quality improvement. Knowing the problems is only half the story.

\section{BACKGROUND}

Well-organised practices deliver higher-quality care. ${ }^{1}$ Evidence-based medicine and the introduction of clinical practice guidelines have been influential, particularly if linked to target setting. However, there has been very little emphasis on process management or cultural change methods to help primary care organisations achieve quality improvement. No attempt has been made to achieve organisational comparisons-as a stimulus for further improvement-at an international level. There is, however, evidence of pressure on primary care organisations to be accountable to external bodies, such as the Quality Outcomes Framework in the $\mathrm{UK}^{2}$ or to meet accreditation standards. ${ }^{3}$ However, most of these methods are summative exercises. The methods also either tend to be automated (coded computer data) or require collection by trained external agents (eg, practice visits $\left.^{4}\right)$. There are very few methods which provide formative assessments and which aim to support practice development and the generation of iterative action plans. ${ }^{5}$ An exception is the Quality Team Development (QTD) method, developed by the Royal College of General Practitioners in the UK. ${ }^{6}$ However, the OTD approach has a high cost, requires multiple meetings with trained facilitators and is labour-intensive. ${ }^{7}$ Moreover, despite increasing interest in achieving international comparisons, efforts to facilitate practice development in primary care have, apart from the European Practice Assessment, ${ }^{4}$ been country-specific.

Primary care organisations have not traditionally invested effort in organisational development: there are many competing priorities. An efficient yet effective approach to practice assessment is needed. $^{8} 9$ In response, we have developed an approach known as the Maturity Matrix ${ }^{10} 11$ which also aims to promote communication and learning. $^{12} 13$ The method involves a facilitator visiting a practice in order to assess the achieved degree of organisational development. The facilitator convenes a multidisciplinary group meeting, typically involving three to 10 individuals. Each individual is asked to complete the Maturity Matrix instrument-without conferring. When 
individuals have completed their assessment, the facilitator leads a discussion where participants compare their evaluations, aiming to arrive at a consensus in a group discussion of approximately $90 \mathrm{~min}$. A consensus score is agreed and the data entered onto an online database. The scores enable the identification of priorities and support the creation of a practice action plan. The UK Maturity Matrix (2003) was evaluated ${ }^{10}$ and adapted for international use (MS Buch, A Adrian Edwards, T Eriksson, submitted, 2009) in a series of evaluations in Slovenia, Switzerland, The Netherlands and Germany ${ }^{10} \quad 11 \quad 14 \quad 15$ and Denmark (MS Buch, A Adrian Edwards, T Eriksson, Submitted, 2009; T Eriksson, VD Siersma, L Løgstrup, et al, Submitted, 2009). ${ }^{16}$

However, concerns were noted, when the UK Maturity Matrix was used in other countries about dimensions choice and the lack of an underpinning theoretical model, that the scale development had not ensured that each step required the completion of the former step, ${ }^{14} 1517$ that is that items had an incremental sequence (a design known as a Guttman scaling ${ }^{18}$ ). We decided to develop a new version of the Maturity Matrix instrument which would be underpinned by a theoretical model and designed for international use. Our literature review had confirmed the need for this approach. ${ }^{8} 9$ The aim of our work was to design this instrument and to evaluate the feasibility of this approach in different countries by forming a collaboration with the European Association for Quality in Family Medicine (EQuiP).

\section{METHODS \\ Development of the International Family Practice Maturity Matrix (IFPMM)}

The development of the IFPMM (http://www.maturitymatrix. co.uk) took place in three phases:

1. selection of organisational dimensions;

2. development of incremental scales;

3. testing the feasibility of the IFPMM.

\section{Selection and refinement of organisational dimensions}

A literature review of organisational factors and frameworks related to high-quality care primary care was conducted ${ }^{12} 1319-22$ and presented to a workshop of EQuiP delegates (Turkey, 2006) in order to generate a list of organisational factors. In each country, a principal investigator (PI) was asked to form an expert panel (10 individuals) of primary care practitioners (clinical or managerial) who had experience of evaluation. The members were asked to use an online system to rank the organisational factors in order of importance to the delivery of high-quality care. These ranked factors formed the basis for the IFPMM dimensions. This process ensured fidelity to organisational issues, and not to the performance of individual clinicians, and that dimensions could be applicable in different healthcare settings.

\section{Development of incremental scales based on a recognised theoretical framework}

Instrument development followed the following steps: alignment to theory, prototype generation and refinement by online consultation with the country-specific panels. We noted that the Manchester Patient Safety Framework (MAPSAF) ${ }^{23}$ had used a similar approach to the Maturity Matrix to assess patient safety. MAPSAF had adapted Westrum's work on the typology of organisational culture: a three-level classification. ${ }^{24}$ At the lowest level, labelled 'pathological,' organisations are power-oriented and demonstrate low cooperation and poor communication, where failure leads to blame, and innovation is discouraged. At the second 'bureaucratic' level, organisations are rule-based and have modest levels of cooperation and communication, failure is analysed, and innovation proposals require formal approval. At the third 'generative' level, organisations are orientated around high performance, cooperation and communication, failure leads to constructive enquiry, and innovation is valued as having potential for improvement.

We used this model as the basis for instrument development by ensuring that the dimensions and items were stratified attributed to the Westrum model. The final English (see figure 1) version was translated using two independent forward-translations, followed by adjustment to country-specific settings and, finally, two independent backward translations, and agreement of a final version. ${ }^{25}$

Testing the feasibility of the IFPMM: an international approach Members of EQuiP were invited to become PIs in an implementation feasibility study and to recruit a minimum of five practices that provided routine primary care services. In a workshop, training materials (handbook, DVD and web-based materials) were provided. Practices were recruited by using an initial familiarisation meeting, followed by a consensus meeting, where the IFPMM assessment was completed and, finally, a feedback meeting.

\section{IFPMM evaluation}

Questionnaires were used to evaluate the IFPMM Consensus meeting process and its contribution to practice development, the skills of the facilitator and views about the overall process. In addition, MJB conducted semistructured telephone interviews, covering the following issues: advantages and disadvantages of using the IFPMM process, any difficulties encountered and suggestions for improvement. The interviews were recorded and transcribed, and a thematic content analysis was performed.

The IFPMM scores were entered into an online database. The automated output (see figure 2) provides a visual representation of the practice score and two comparative benchmarks: (1) the average practice score benchmark (a mean of all other practice assessments) and (2) comparison with organisations with scores at the leading edge score benchmark (ie, the aggregated top $25 \%$ ). Dimension scores were rescaled (0 to 100), with the IFPMM global score being the average of the seven dimension scores, and score variation within and between countries was examined. Dimension cohesiveness was assessed by Spearman correlations, on an intercountry (ecological) basis using country means and on an intracountry basis by calculating residuals.

\section{RESULTS}

\section{Selection and refinement of organisational dimensions}

The literature review provided evidence that the following changes made a difference to patient care: the implementation of guidelines and recall systems, increasing consultation length and the use of targeted incentive systems: ${ }^{19}$ with this input, the workshop generated a list of 69 factors. We decided that the most appropriate organisational framework was the Dutch adaptation of the European Framework for Quality Management (EFOM): ${ }^{20}$ it is divided into the following aspects: leadership, policy and strategy, people management, resources, processes, appreciation by customers, by people and by society, and lastly, business results. The brainstorm factors were organised according to this framework and ranked by the 
International Family Practice Maturity Matrix www.maturitymatrix.co.uk version 2.0 June 2007

(1)

Figure 1 International Family Practice Maturity Matrix, copyright-free.

country-specific panels (Austria, Belgium, Czech Republic, Denmark, Finland, Ireland, The Netherlands, Norway, Poland, Portugal, Slovenia, Spain, Sweden, Turkey and the UK). Full results are published elsewhere. ${ }^{19}$

The IFPMM dimensions were based on the highest ranked aspects. However, although highly ranked, the financial management and leadership dimensions were excluded on the grounds that it is not possible to debate financial management in a group where discussion about profit sharing would not be possible and, similarly, that a debate on leadership would be too contentious. Two aspects, 'Appreciation by staff' and 'People management,' were merged into a dimension called 'Managing staff.' table 1 summarises the process of arriving at the final seven dimensions included in the IFPMM.

\section{Development of incremental scales}

The development of the IFPMM instrument involved five iterations. Feedback from the EQuiP workshop in Barcelona (2006) suggested shorter item phrasing, and 120 (75\%) of the 159 panel members participated. There was a high agreement (82\%) that the items could be applied in each country. Thirteen translations were produced (Albanian, Croatian, Dutch, French (Walloon), German, Greek, Italian, Norwegian, Portuguese, Slovene, Spanish, Swedish and Turkish).

Testing the feasibility of the IFPMM: an international approach At the end of a 12-month feasibility study, data were available from 73 practices in 12 countries: Belgium (four practices), Croatia (five), Germany (22), Greece (five), Kosovo (five), Nigeria (six), Norway (four), Portugal (three), Slovenia (five), Spain (five), The Netherlands (five) and the UK (four). Nineteen practices were single-handed; a further 17 had two doctors, and other practices ranged from three to nine practitioners. Only seven practices had no nurse as part of the staff. The PIs in Nigeria (YY), Kosovo (IM) and Germany (CK) joined the study independently of EQuiP.

\section{IFPMIM evaluation}

The evaluation of the IFPMM consensus meeting process was positive: there was a high agreement from 70 practices that there was sufficient time to discuss organisational development (99\%), that the process facilitated talk about organisational development $(90 \%)$, that respondents would like to use the IFPMM again (97\%) and that the comparative benchmark was useful (94\%). Similarly, positive evaluations of the facilitator were received from 70 practices: that the process was effective (99\%), that clear aims were stated (90\%) and that there was a willingness to recommend the facilitator to others $(99 \%)$. Positive evaluations were received for the identification of improvement priorities (97\%) and that the process added value (95\%). In summary, the evaluation confirmed that the IFPMM was viewed as a valuable method for assessing organisational development in primary care across a range of settings. The two benchmarks were considered to be a novel and useful way to motivate quality improvement.

A total of 14 individuals from Belgium, Croatia, Germany, Greece, The Netherlands, Nigeria, Portugal, Norway, UK, Slovenia and Spain were interviewed using a semistructured 


\section{International Family Practice Maturity Matrix Benchmark example
Key $X X X X X X=$ Current Results $-12 / 09 / 2008$
$==-$ Benchmark Average
$=\because n=$ Leading Edge

Based on 71 practices

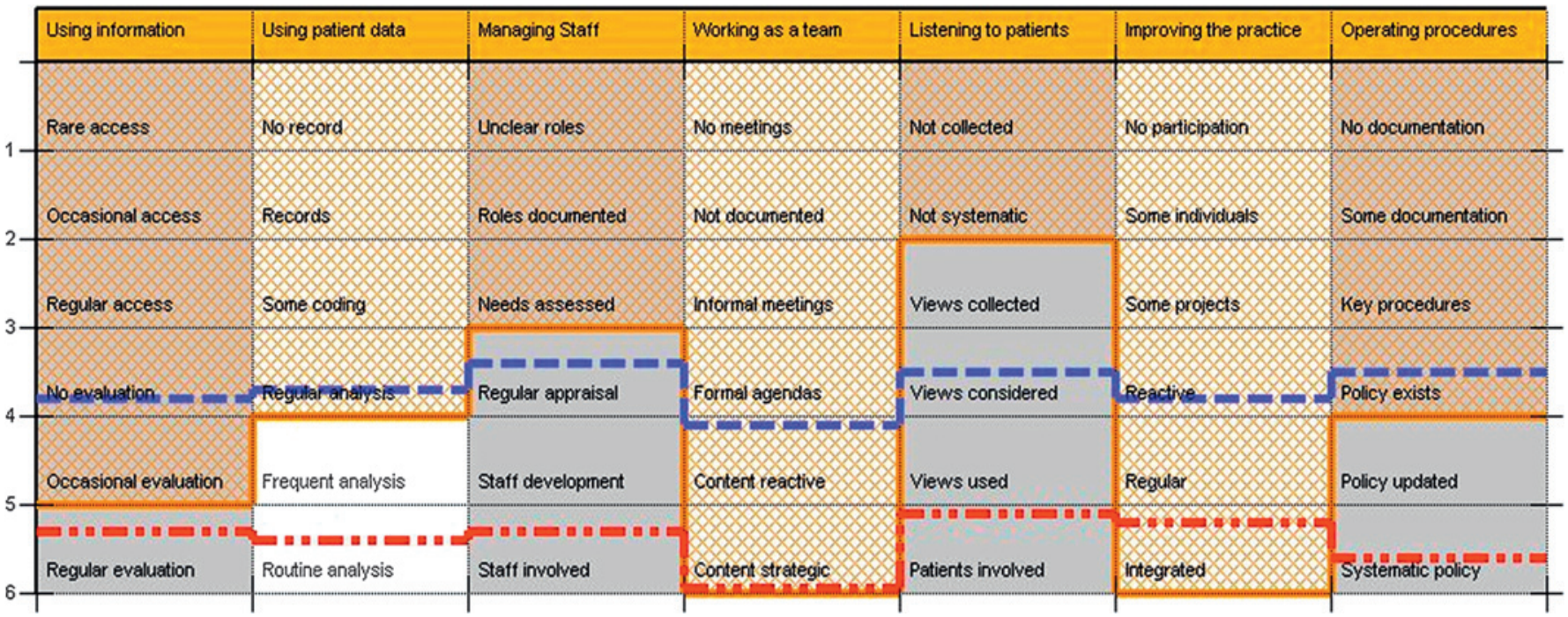

Figure 2 International Family Practice Maturity Matrix consensus matrix showing practice score, average and leading edge benchmark.

schedule. Thematic analysis showed that the method, the clarity of the instrument design and the team-building group process aspects were consistently appreciated. In addition, the benchmarking feature helped prioritise areas for change and generate enthusiasm for action. There were concerns that the assessment process requires adaptation for use in very large or solo practices. The interviewees also felt that the IFPMM process needs to be embedded in an organisational development system where support for change management should be linked to wider system of quality improvement at a group or regional level.

\section{FPMM scores and comparative benchmarks \\ IFPMM scores}

Note that practice samples are small and cannot be representative because participation was voluntary and by invitation Nevertheless, the global IFPMM scores had a wide range across

Table 1 Results of the ranking process and dimension ranking and refinement

\begin{tabular}{|c|c|c|c|}
\hline Subaspects (not in rank order) & Phase 1: Top 10 dimensions & Phase 2: Selecting dimensions & $\begin{array}{l}\text { Phase 3: Refining terminology for } \\
\text { International Family Practice Maturity } \\
\text { Matrix }\end{array}$ \\
\hline 1. Process: innovation and creativity & Innovation & $\begin{array}{l}\text { Developing the practice by improving the } \\
\text { process of care: audits, quality } \\
\text { improvement cycles }\end{array}$ & Improving the practice \\
\hline $\begin{array}{l}\text { 2. Processes: systematic process } \\
\text { management }\end{array}$ & Standard operating procedures & $\begin{array}{l}\text { Operating procedures, that is documented } \\
\text { standardised steps for organisational and } \\
\text { care processes }\end{array}$ & Operating procedures \\
\hline $\begin{array}{l}\text { 3. Resources: technology and knowledge } \\
\text { management }\end{array}$ & Internal knowledge management & $\begin{array}{l}\text { Using patient data: information recorded } \\
\text { from consultations. Coding and analysis } \\
\text { potential. }\end{array}$ & Using patient data \\
\hline $\begin{array}{l}\text { 4. Resources: technology and knowledge } \\
\text { management }\end{array}$ & External knowledge management & $\begin{array}{l}\text { Using information: accessing evidence } \\
\text { about best practice, sharing the evidence } \\
\text { and assessing its applicability and quality }\end{array}$ & Using information \\
\hline 5. Appreciation by society & Client (patient) focus & $\begin{array}{l}\text { Listening to patients: their views and } \\
\text { experiences and complaint process }\end{array}$ & Listening to patients \\
\hline 6. Policy and strategy: Communication & Team working processes & $\begin{array}{l}\text { Working as a team: organisation-wide } \\
\text { communication, using meetings, minutes, } \\
\text { and documented action }\end{array}$ & Working as a team \\
\hline $\begin{array}{l}\text { 7. Appreciation by staff and people } \\
\text { management: planning }\end{array}$ & Human resource management & $\begin{array}{l}\text { Managing staff: recruitment processes, } \\
\text { role clarity, staff development and } \\
\text { appraisal }\end{array}$ & Managing staff \\
\hline 8. Resources: financial management & Resource management & \multicolumn{2}{|c|}{$\begin{array}{l}\text { These subaspects were excluded as International Family Practice Maturity Matrix } \\
\text { dimensions: financial details are difficult to confront in open discussion where staff have } \\
\text { different roles (eg, employees as well as employers), and similarly, detailed discussion } \\
\text { about leadership in a group setting may be contentious and is best postponed, if this needs } \\
\text { attention }\end{array}$} \\
\hline 9. Leadership: visible commitment & Leadership & & \\
\hline 10. Policy and strategy: leadership & Leadership & & \\
\hline
\end{tabular}


Table 2 Maturity Matrix Global Scores (all practices, country level)

\begin{tabular}{lrrrll}
\hline Country & N & Mean & SD & Minimum score & Maximum score \\
\hline Belgium & 4 & 60.7 & 13.3 & 48.6 & 77.1 \\
Croatia & 5 & 35.4 & 4.8 & 31.4 & 42.9 \\
Germany & 22 & 64.4 & 19.2 & 28.6 & 94.3 \\
Greece & 5 & 49.1 & 17.1 & 34.3 & 77.1 \\
Kosovo & 5 & 40.6 & 14.3 & 25.7 & 62.9 \\
The Netherlands & 5 & 61.1 & 17.8 & 34.3 & 80.0 \\
Nigeria & 6 & 21.0 & 4.7 & 14.3 & 28.6 \\
Norway & 4 & 57.9 & 15.2 & 40.0 & 74.3 \\
Portugal & 3 & 60.0 & 24.9 & 31.4 & 77.1 \\
Slovenia & 5 & 63.4 & 14.3 & 45.7 & 80.0 \\
Spain & 5 & 33.7 & 11.3 & 20.0 & 48.6 \\
UK & 4 & 79.3 & 7.1 & 71.4 & 88.6 \\
Total & 73 & 53.9 & 21.2 & 14.3 & 94.3 \\
\hline
\end{tabular}

the 73 practices, from 14 to 94 . There was significant variation $(\mathrm{p}<0.001)$ in the mean global scores from the 12 countries, from 21 (Nigeria) to 79 (UK); see table 2 and figure 3. Rank correlations between scores for the seven dimensions ranged from 0.4 to 0.7 (median 0.5). Broadly similar correlations were obtained from residuals ( 0.3 to 0.7 , median 0.4 ) and on an ecological basis ( 0.2 to 0.8 , median 0.6 ), indicating that the moderately large positive correlations between the dimensions are driven by both country- and practice-level factors.

\section{Average and leading edge benchmark}

Figure 2 illustrates the IFPMM output. The yellow line provides the consensus IFPMM score for the practice. The blue line shows the average benchmark. The red line illustrates the leading edge benchmark.

\section{DISCUSSION}

This study demonstrates the feasibility of developing an organisational assessment tool for primary care organisations. We believe the assessment is valid at the organisational level and leads to improvements in annual timescales: it also has the potential to create valuable comparison data where it is appropriate to examine similar structures and processes, and the process can be applied across a range of health settings. It was possible to introduce this assessment method in 11 countries in Europe and one in Africa, and to generate comparison benchmarks based on the data collected.
We caution against intercountry comparisons, however: the samples are too small, voluntary and therefore subject to selection bias. There are also local, regional and contextual factors that need to be taken into account in order to interpret the results. In ideal circumstances, further training of the facilitators would ensure greater assessment consistency. Nevertheless, evaluations of the assessment process were uniformly positive: participants felt that the approach efficiently identifies organisational development priorities and motivates efforts to achieve change, especially at the practice level.

The Maturity Matrix concept was initiated in 1996, was the subject of a doctorate thesis, ${ }^{17}$ and was adapted for use in Denmark (MS Buch, AA Edwards, T Eriksson, Submitted, 2009; T Eriksson, VD Siersma, L Løgstrup, et al, submitted, 2009). ${ }^{16}$ Criticism of the initial version led to this study in which a new instrument was developed for international use. We are not aware of any comparable work in primary care. In terms of research methods, we would have preferred to assess more practices and for facilitators to have had more training (MS Buch, AA Edwards, T Eriksson, submitted, 2009). ${ }^{11}$ We attempted to include practices from the US and Canada, in order to enhance the validity, and hope that this will take place in future studies.

It is important to note that the aim was not to create a summative measure or a 'league table' of practice quality but to generate an assessment, albeit 'a soft fuzzy' assessment, that engages participating practices and enables comparisons against peers using the concept of benchmarks without confronting sensitive issues such as financial issues in group settings.

\section{Findings in context}

We could not identify similar studies. Most assessment methods in this context have used a checklist approach to provide external assessments ( $\mathrm{Y}$ Engels, S Campbell, M Dautzenberg, et al, submitted, 2004) ${ }^{8}$ often aligned to accreditation efforts. ${ }^{3}$ A notable exception is the QTD method developed by the RCGP, which is a team-based formative assessment. Macfarlane's in-depth interview study noted that, although the process was valued, it required significant additional resources, and the assessment is not widely implemented. ${ }^{7}$ Work in Denmark, on an adapted version of the UK Maturity Matrix, reports that participants were positive about the facilitated group-based assessment approach (MS Buch, AA Edwards, T Eriksson,
Figure 3 Consensus matrix: global and dimension scores.

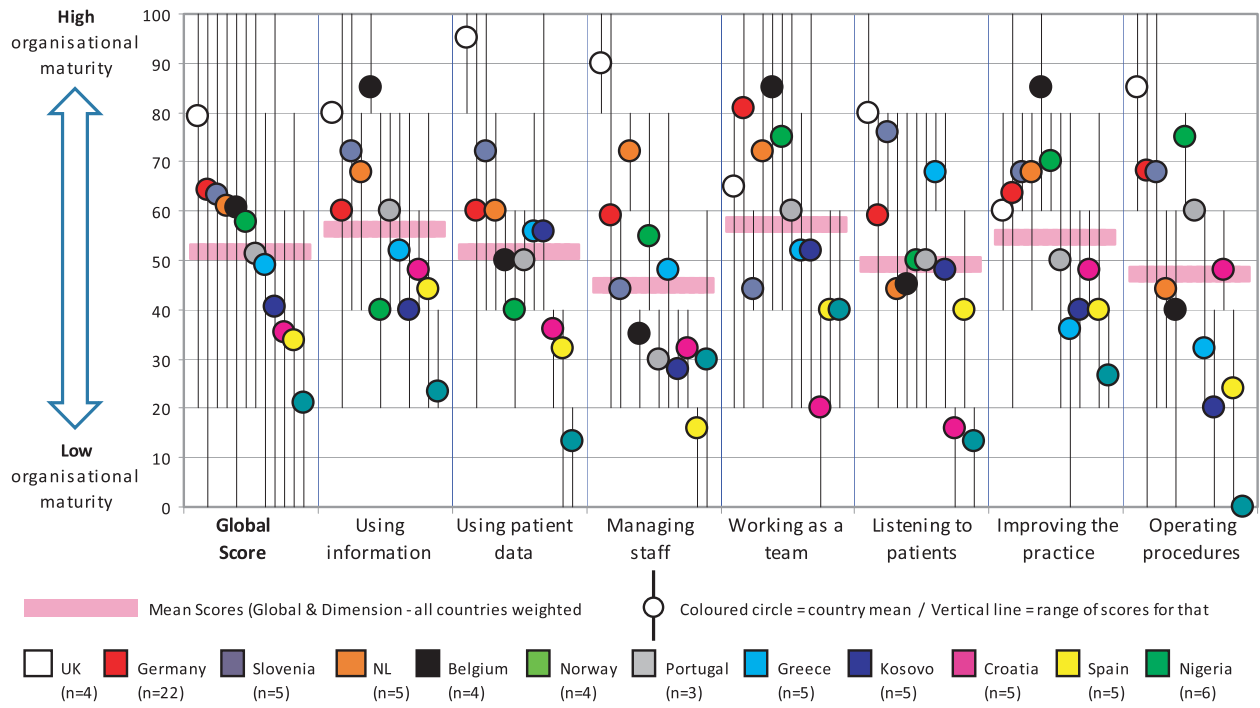


submitted, 2009; T Eriksson, VD Siersma, L Løgstrup, et al, submitted, 2009). ${ }^{16}$

\section{CONCLUSIONS}

We are not aware of any other organisational assessment methods for primary care that have an international aim or that have used an organisational framework and a theoretical model to guide their development. We recognise that formal validation has not been achieved, but the purpose is not to achieve high reliability: the principal aim is to achieve an organisational assessment that is capable of identifying priorities for change and at the same time motivating the participants to take action. We think it may be possible to generate a generic version that could be used in organisational unit that Batalden refers to as microsystems. ${ }^{26}$ However, our next aim is to achieve wider implementation and to ensure linkages are made between the IFPMM results and planned investment in quality improvement. Knowing the problem is only half the story: improving the organisation so that the problems are tackled and managed is the more difficult task, especially when this involves changing cultures and communication processes as well as introducing management tools such as a documented interdisciplinary meetings and developing standardised operating procedures. An international database of practice-based assessments is being developed, and we would welcome initiatives to join this effort.

\section{Author affiliations}

'Department of Primary Care and Public Health, School of Medicine, Cardiff University, Cardiff, UK

${ }^{2}$ School of Psychology, University of Southampton, Southampton, UK

${ }^{3}$ Department of General Practice, Centre of Health and Society, University of Copenhagen, Copenhagen, Denmark

${ }^{4}$ Scientific Institute for Quality of Healthcare, Radboud University Nijmegen Medical Centre, Nijmegen, The Netherlands

${ }^{5}$ Department for Medical Sociology, University of Cologne, Cologne, Germany

${ }^{6}$ Department of Family Medicine, 'Andrija Stampar' School of Public Health, Medical School University of Zagreb, Zagreb, Croatia

${ }^{7}$ Institute of Child Health \& Primary Care, College of Medicine, Lagos, Nigeria

${ }^{8}$ Department of Family Practice, Medical Faculty, Ljubliana, Slovenia

${ }^{9}$ Netherlands College of General Practitioners, Utrecht, The Netherlands

${ }^{10}$ Greek Association of General Practice, Quality Assurance Committee, Athens,

Greece

${ }^{11}$ Société Scientifique de Médecine Générale (SSMG), Brussels, Belgium

${ }^{12}$ Family Medicine Center, Mitrovica, Kosovo

${ }^{13}$ Mission for the reform of Primary Health Care, Ministry of Health, Rua Gomes, Freire, Lisbon, Portugal

${ }^{14}$ National Centre for Emergency Primary Health Care, Bergen, Norway

${ }^{15}$ Grupo Aragonés de Investigación en Atención Primaria, Aragon Health Sciences Institute, Alcañiz, Spain

${ }^{16}$ Lessius University College, Department of Applied Psychology, Antwerp, Belgium

Acknowledgements We thank the European Association for Quality in General Practice/Family Medicine (EQuiP) for their support and cooperation and EQuiP delegates, R Glehr, L Seuntjes, B Seifert, T Eriksson, E Ketola, M O'Riordan, K in't Veld, J Thesen, ZJ Król, L Pisco, J Kersnik, JM Turón, P Stenström and N Dağdeviren for convening the country-specific panels. Most of the Pls in the feasibility study were recruited from EQuiP ranks. In addition to already named authors, we are grateful to the following Pls: T Schofield (UK) and S Uka (Kosovo). Full translations for the IFPMM instrument were achieved by all named Pls as well as by A Campanini (Italy), P Stenström (Sweden) and N Daĝdeviren (Turkey). In addition to Pls conducting consensus meetings, IFPMM facilitation was provided by K Knops (Belgium), J Warns and K Pralle (Germany), K Prestegaard and J Falhof (Norway), H Covita (Portugal), B Oliván and Y Lopez (Spain), and L Roebroeck-van de Zande (The Netherlands). Finally, we wish to acknowledge the support of participating practices, Oscom Solutions (database design) and the support provided by E Hussein, S Evans and A Watkins. EQuiP (http://www.equip.ch/) is a constituent part of the World Organization of Family Doctors (WONCA), and the members have expertise in quality improvement in primary care.

Funding This study was achieved without external funding by collaboration between Radboud University Nijmegen Medical Centre and Cardiff University. Other Funders: internal.
Competing interests The IFPMM instrument is available copyright-free. A licence is required to gain access to other IFPMM resources and the benchmark database, under the administration of Cardiff University on a not-for-profit basis. No author benefits financially from the licensing arrangements.

Contributors GE developed the Maturity Matrix method and, with AE and LT, designed the IFPMM study. GE, AE and LT, together with the Steering Group (RG, JB, TE, CK), managed the development and implementation of the IFPMM. LT, M-JB and the IFPMM Steering Group were involved in the production of the final report. RN analysed the data; RR produced the graphic. The principal investigators were responsible for implementing the IFPMM in their own countries. GE and M-JB wrote the article, with significant contributions from LT and AE. All authors have been involved in editing the manuscript and have agreed the final version.

Provenance and peer review Not commissioned; externally peer reviewed.

\section{REFERENCES}

1. Campbell SM, Roland MO, Middleton E, et al. Improvements in quality of clinical care in English general practice 1998-2003: Iongitudinal observational study. BMJ 2005; 331:1121.

2. Doran T, Fullwood C, Gravelle $\mathrm{H}$, et al. Pay-for-performance programs in family practices in the United Kingdom. N Engl J Med 2006:355:375-84.

3. Buetow SA, Wellingham J. Accreditation of general practices: challenges and lessons. Oual Saf Health Care 2003:12:129-35.

4. Engels Y, Dautzenberg M, Campbell S, et al. Testing a European set of indicators for the evaluation of the management of primary care practices. Fam Pract 2006;23:137-47.

5. Elwyn G, Carlisle $S$, Hocking $P$, et al. Practice and professional development plans (PPDPs): results of a feasibility study. BMC Fam Pract 2001:2:1. http://www. biomedcentral.com/1471-2296/2/1.

6. Royal College of General Practitioners. Quality Team Development (OTD). London: Royal College of General Practitioners, 2003. http://www.rcgp.org.uk/rcgp/ quality_unit/qtd/index.asp.

7. Macfarlane F, Greenhalgh T, Schofield T, et al. The RCGP Quality Team Development Programme: a qualitative evaluation. Qual Saf Health Care 2004;13:356-62.

8. Rhydderch M, Engels $Y$, Edwards A, et al. Organisational assessment in general practice: a systematic review and implications for quality improvement. J Eval Clin Pract 2005; 11:366-78.

9. Rhydderch M, Elwyn G, Marshall M, et al. Organisational change theory and the use of indicators in primary care organisations. Qual Saf Health Care 2004;13:213-17.

10. Elwyn G, Rhydderch M, Edwards A, et al. Assessing organisational development in primary medical care using a group based assessment: the Maturity Matrix. Qual Saf Health Care 2004;13:287-94.

11. Rhydderch $\mathbf{M}$, Edwards A, Marshall $\mathbf{M}$, et al. Developing a facilitation model to promote organisational development in primary care practices. BMC Fam Pract 2006:7:38.

12. Ferlie EB, Shortell SM. Improving the quality of health care in the United Kingdom and the United States: a framework for change. Milbank Q 2001;79:281-315.

13. Koeck C. Time for organisational development in healthcare organisations. BMJ 1998;317:1267-8.

14. Rhydderch $\mathbf{M}$, Edwards A, Marshall $\mathbf{M}$, et al. Maturity Matrix: A criterion validity study of an instrument to assess organisational development in European general practice. Qual Prim Care 2006;14:33-43.

15. Edwards A, Rhydderch M, Engels $Y$, et al. Assessing organisational development in Euorpean primary care using a group based method: the Maturity Matrix (Version 2003). Int J Qual Health Care In Press.

16. Løgstrup L, Edwards A, Waldorff FB, et al. GP and staff evaluation of the Maturity Matrix as a tool to assess and improve organisational development in primary care. Int J Health Care Oual Assur 2009;22:686-700.

17. Rhydderch M. Assessing organisational development in family practice: the Maturity Matrix. PhD thesis. Nijmegen: Radboud University Nijmegen, 2006.

18. Guttman L. The basis for scalogram analysis. In: Stouffer A, ed. Measurement and Prediction. The American Soldier vol IV. New York: Wiley, 1950.

19. Tapp L, Bekkers M, Braspenning J, et al. Developing the International Family Practice Maturity Matrix, an organisational assessment tool for primary care. Cardiff: Cardiff University.2009. ISBN: 978-0-9550975-3-9.

20. Nabitz UW, Klazinga NS. The European Framework for Quality Management (EFOM) approach and the Dutch Quality Award. Int J Health Care Oual Assur 1999:12:65-70.

21. Grol R, Baker R, Moss F. Quality improvement research: understanding the science of change in health care. London: BMJ Books, 2004.

22. Grol R, Wensing R, Eccles M. Improving patient care. The implementation of change in clinical practice. Edinbrugh: Elsevier, 2005

23. Ashcroft DM, Morecroft C, Parker D, et al. Safety culture assessment in community pharmacy: development, face validity, and feasibility of the Manchester Patient Safety Assessment Framework. Qual Saf Health Care 2005;14:417-21.

24. Westrum R. A typology of organisational culture. Qual Saf Health Care 2004;13:22-7.

25. Beaton DE, Bombardier C, Guillemin F, et al. Guidelines for the process of crosscultural adaptation of self-report measures. Spine 2000;25:3186-91.

26. Nelson EC, Godfrey MM, Batalden PB, et al. Clinical microsystems, part 1. The building blocks of health systems. Jt Comm J Qual Patient Saf 2008;34:367-78. 